\title{
Detection of Substantia Nigra Echogenicities in 3D Transcranial Ultrasound for Early Diagnosis of Parkinson Disease
}

\author{
Olivier Pauly ${ }^{1,2}$, Seyed-Ahmad Ahmadi ${ }^{2}$, Annika Plate ${ }^{3}$, \\ Kai Boetzel ${ }^{3}$, and Nassir Navab ${ }^{2}$ \\ 1 Institute of Biomathematics and Biometry, Helmholtz Zentrum München, Germany \\ ${ }^{2}$ Computer Aided Medical Procedures, Technische Universität München, Germany \\ 3 Department of Neurology, Ludwig-Maximilians-University of Munich, Germany \\ \{pauly, ahmadi, navab\}@cs.tum.edu
}

\begin{abstract}
Parkinson's disease (PD) is a neurodegenerative movement disorder caused by decay of dopaminergic cells in the substantia nigra (SN), which are basal ganglia residing within the midbrain area. In the past two decades, transcranial B-mode sonography (TCUS) has emerged as a viable tool in differential diagnosis of $\mathrm{PD}$ and recently has been shown to have promising potential as a screening technique for early detection of $\mathrm{PD}$, even before onset of motor symptoms. In TCUS imaging, the degeneration of SN cells becomes visible as bright and hyper-echogenic speckle patches (SNE) in the midbrain. Recent research proposes the usage of 3D ultrasound imaging in order to make the application of the TCUS technique easier and more objective. In this work, for the first time, we propose an automatic 3D SNE detection approach based on random forests, with a novel formulation of SNE probability that relies on visual context and anatomical priors. On a 3D-TCUS dataset of 11 PD patients and 11 healthy controls, we demonstrate that our SNE detection approach yields promising results with a sensitivity and specificity of around $\mathbf{8 3 \%}$.
\end{abstract}

\section{Introduction and Medical Motivation}

Parkinson's Disease (PD) is a neuro-degenerative movement disorder which has been the matter of increasing research in the medical and scientific community for the past decades. The primary symptoms of PD affect the motoric system, such as rigidity, shaking or slowness, but PD may also evoke non-motor symptoms such as dementia in later stages of the disease. The root cause of PD is the death of dopaminergic substantia nigra (SN) cells, which are located in the midbrain area. Although it is not known whether it is the cause or an effect of SN cell death, the progress of the disease is accompanied by a build-up of ferrite deposits within the SN. Over the past two decades, several studies have shown that these physiological changes can be visualized using transcranial ultrasound (TCUS), making this imaging technique a viable tool in differential diagnosis of PD [12. Additionally, it has been shown recently that TCUS can be used as an early indicator of PD [2]. This result is particularly relevant, since

N. Ayache et al. (Eds.): MICCAI 2012, Part III, LNCS 7512, pp. 443-450, 2012.

(C) Springer-Verlag Berlin Heidelberg 2012 
it increases the hope that TCUS can be used as a cheap, quick and non-invasive early-detection and screening tool for large populations. The changes in SN are visible in TCUS in form of hyper-echogenicities, i.e. small bright speckle patches, within the midbrain. If performed by an expert sonographer with substantial experience in this technique, sensitivity and specificity of this technique can be as high as $90 \%$ [7]). However, the challenging nature of TCUS images causes high intra- and inter-rater variability and makes it difficult for less experienced groups to reach the diagnostic reliability of expert groups in this field [11. Recently, the usage of three-dimensional (3D-) TCUS started being investigated for PD diagnosis, since it can make this promising PD screening technique easier, more objective, and more significant due to the volumetric analysis of substantia nigra echogenicities (SNE). In this paper, we introduce a fully automatic approach for the detection of SNE voxels within the midbrain, once the latter has been localized. There is little related work in literature concerning the automatic analysis of SNE, but similar to our work, all approaches we are aware of perform a midbrain ROI segmentation first and a SNE detection within the midbrain subsequently. Kier et al. 9] and Chen et al. 4. respectively perform SN pixel detection using morphological operators or image-feature-based SVM classification, both within a manually segmented midbrain in 2D. Engels et al. 6] use a hierarchical finite-element model and active contours to simultaneously segment the midbrain and SNEs in 2D. Despite early work on segmentation of midbrain area in 3D ultrasound [1, to our knowledge, there is no previous work on (semi-) automatic SNE analysis in 3D-TCUS. The main contributions of this paper are therefore to 1) propose a novel and volumetric SNE detection method based on random-forest, 2) formulate a detection paradigm mimicking human experts by using probabilistic modeling of visual and spatial SNE features and 3) show the reliability of our SNE detection approach on a set of 3D-TCUS volumes from 22 subjects.

\section{Materials and Methods}

As illustrated by Fig 1, an experimented observer can detect PD-related hyperechogenicities in the left and right SN using 3D-TCUS. Unfortunately, TCUS cannot visualize the SN regions themselves, but only the high-contrast SNE speckles located randomly within the area of SN. Thus, relying on prior knowledge of the midbrain anatomy and the known rough location of the SN within the midbrain, an experimented observer has to decide whether an echogenicity belongs to the SN or not based on location and intensity of speckle patches. This makes the detection of Parkinson-related SNEs quite challenging. In the present work, we aim at providing a reliable detection of PD-related SNEs in 3D by analoguously integrating two types of information: (i) visual context and (ii) spatial location within the midbrain.

Problem Statement: Let us consider an intensity function denoted by $\mathbf{I}$ : $\Omega \rightarrow \mathbb{R}$, where $\Omega \subset \mathbb{R}^{3}$ is the image domain representing the $3 \mathrm{D}$ ultrasound 

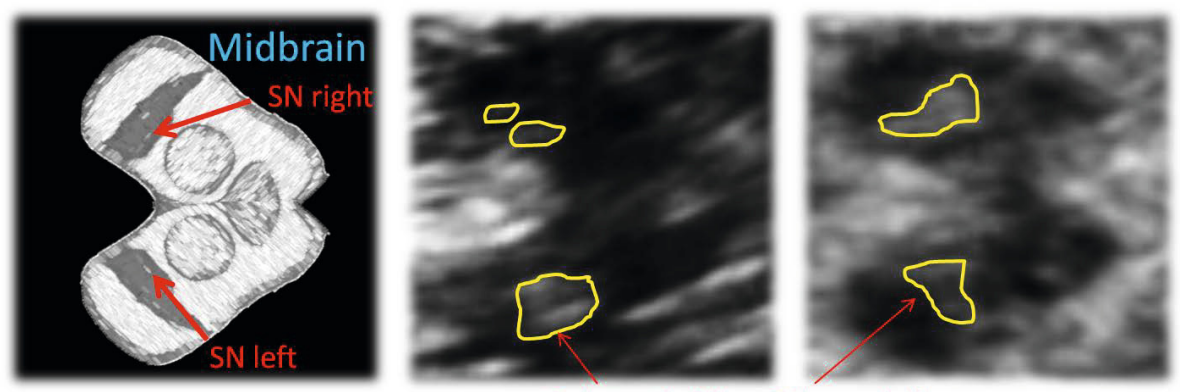

Substantia Nigra Echogenicities

Fig. 1. Goal of our approach: On the left, the anatomy of the midbrain is detailed, showing the Substantia Nigra regions located at the front of both hemispheres. The other images show examples of typical SNE speckle patterns (in yellow) in 3D-TCUS transversal slices.

data. We further assume that we are given a segmentation of the midbrain $\mathcal{M} \subset \Omega$, either from a manual expert segmentation or alternatively from the output of a ROI detection algorithm 11. In this paper, we propose to formulate the detection problem as a classification task in which each voxel $\mathbf{x} \in \mathcal{M}$ needs to be associated to a label $\mathbf{c} \in\{0,1\}$, where 0 denotes the background and 1 the Substantia Nigra Echogenicities (SNE) class. In fact, $\mathbf{c}$ is the realization of 2 random variables $(\mathcal{E}, \mathcal{S})$ where $\mathcal{E}$ represents the observation of an echogenicity and $\mathcal{S}$ of the Substantia Nigra $(\mathrm{SN})$, i.e. $\mathbf{c}=1$ if and only if $\mathcal{E}=1$ and $\mathcal{S}=1$. Therefore, we aim at learning $P(\mathcal{E}, \mathcal{S} \mid \mathbf{x}, \mathbf{I})$, which represents the joint probability of observing an echogenicity $\mathcal{E}$ belonging to the $\mathrm{SN} \mathcal{S}$ given the location $\mathbf{x}$ and the intensity function $\mathbf{I}$. It is important to note that (1) it is not the SN itself which causes hyper-echogenicities but only potential acoustic micro-scatterers residing within it and (2) echogenicities can happen in the whole skull in TCUS due to tissue boundaries and micro-scatterers present in the entire brain tissue. Hence, we can assume the independence of the random variables $\mathcal{E}$ and $\mathcal{S}$, and decompose this joint probability as follows:

$$
P(\mathcal{E}, \mathcal{S} \mid \mathbf{x}, \mathbf{I})=P(\mathcal{E} \mid \mathbf{x}, \mathbf{I}) P(\mathcal{S} \mid \mathbf{x})
$$

The first term $P(\mathcal{E} \mid \mathbf{x}, \mathbf{I})$ is a data term, encoding the probability of observing an echogenicity given some visual information at location $\mathbf{x}$, and the second term $P(\mathcal{S} \mid \mathbf{x})$ is an anatomical prior not depending on $\mathbf{I}$, i.e. the ultrasound data. As learning these probability distributions is challenging due to the dimensionality of the problem, we propose to use two discriminative models based on random forests. Following a "divide" and "conquer" strategy, random forests [3] provide efficient piecewise approximations of any distribution in high-dimensional spaces by: (1) partitioning the space using simples decisions, and (2) estimating the posterior in each "cell" of this space. As shown in [510], random forests have been successfully applied to the task of multiple organ localization in CT and MR scans. Geremia et al. in [8] demonstrated state-of-the-art results for the 
segmentation of multiple-sclerosis lesions based on multi-channel MRI data. In addition to a forest using visual context, we propose to learn a novel spatial prior based on two hemisphere-specific coordinate systems. In the following, we describe how to use random forests for learning: (1) the data term $P(\mathcal{E} \mid \mathbf{x}, \mathbf{I})$ and $(2)$, the prior $P(\mathcal{S} \mid \mathbf{x})$.

Learning the Data Term $\boldsymbol{P}(\mathcal{E} \mid \mathbf{x}, \mathbf{I})$ : In TCUS, echogenicities are characterized by higher intensities and higher contrast. Therefore, we propose to describe the visual context of a voxel at location $\mathbf{x}$ by extracting a set of simple features that encode the mean intensities in cuboidal regions of different sizes in the neighborhood of $\mathbf{x}$ similarly as in [8]. Let us denote by $\mathcal{X}$ the space spanned by these simple features, and $\mathbf{X}$ the feature representation associated to a voxel at location $\mathbf{x}$. We consider a training set $\left(\mathbf{X}_{n}, \mathcal{E}_{n}\right)_{n=1}^{N}$, where each feature vector $\mathbf{X}_{n}$ is associated to a label $\mathcal{E}_{n}$ which is equal to 1 if there is an echogenicity at location $\mathbf{x}_{n}$ and 0 if not. Consisting of an ensemble of independent trees, a random forest permits to efficiently partition this high-dimensional space $\mathcal{X}$. Each tree can be seen as a directed acyclic graph where each node consists in a decision function $f_{\mathbf{v}, \tau}$ defined as $f_{\mathbf{v}, \tau}(\mathbf{X})=(\mathbf{X} \cdot \mathbf{v} \geq \tau)$. $\mathbf{v}$ is a vector of dimensionality $\operatorname{dim}(\mathcal{X})$ having only 1 non-zero value, and $\tau \in \mathbb{R}$ a threshold. According to the result of this decision function, incoming data are pushed towards the left or right child of the current node. Note that the role of $\mathbf{v}$ is to select a feature dimension where to perform the decision, yielding thus axis-aligned splits in $\mathcal{X}$. Let us denote by $\Delta$ the set of feature points from $\mathcal{X}$ reaching the current node, and $\Delta_{l}, \Delta_{r}$ the subsets respectively sent to the left and right child nodes. At each node, the choice of $\mathbf{v}$ and $\tau$ is optimized following a greedy optimization strategy. A set $\Gamma$ of functions are randomly drawn and the best candidate $\left(\mathbf{v}^{*}, \tau^{*}\right)$ is selected by maximizing information gain:

$$
\left(\mathbf{v}^{*}, \tau^{*}\right)=\underset{(\mathbf{v}, \tau) \in \Gamma}{\operatorname{argmax}}\left(\mathbf{H}(\Delta)-w_{l} \mathbf{H}\left(\Delta_{l}\right)-w_{r} \mathbf{H}\left(\Delta_{r}\right)\right)
$$

where $w_{l}=\left|\Delta_{l}\right| /|\Delta|$ and $w_{r}=\left|\Delta_{r}\right| /|\Delta|$. H corresponds to the classical Shannon's entropy $\mathbf{H}=-\sum_{e \in\{0,1\}} P(\mathcal{E}=e \mid \mathbf{x}, \mathbf{I}) \log (P(\mathcal{E}=e \mid \mathbf{x}, \mathbf{I}))$, the posterior distribution being estimated from the set of points in the current node as:

$$
P(\mathcal{E}=e \mid \mathbf{x}, \mathbf{I})=\frac{\left|\left\{\mathbf{X}_{n} \in \Delta, \mathcal{E}_{n}=e\right\}\right|}{\left|\left\{\mathbf{X}_{n} \in \Delta\right\}\right|}
$$

By optimizing this energy function, the tree aims at minimizing the uncertainty on the random variable $\mathcal{E}$, encouraging thereby the creation of leaves containing either mostly echogenicities, or mostly background. Nodes are grown until a maximal tree depth has been reached, or when the number of feature points falls below a given threshold. Finally, in each leaf, the posterior distribution $P(\mathcal{E} \mid \mathbf{x}, \mathbf{I})$ is computed on the set of features points reaching this leaf using Eq 3 and stored. Now, to predict the probability of observing an echogenicity at a location $\mathbf{x}$ for an unseen ultrasound volume of the midbrain, one just needs to first extract its associated feature vector $\mathbf{X}$, to push it downward the tree until it 


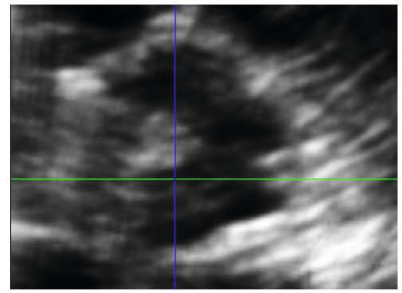

Midbrain in TC-US Transversal plane

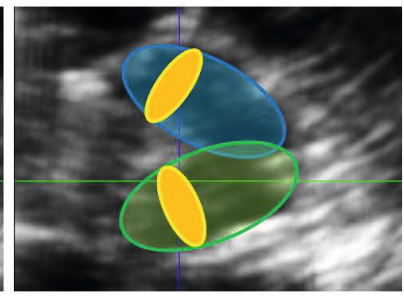

Left hemisphere / Right hemisphere Substantia Nigra

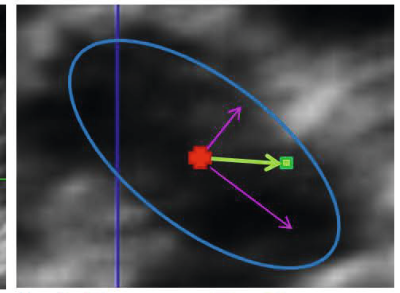

Hemisphere center/Coordinate system Voxel relative coordinates

Fig. 2. Midbrain anatomy: in the transversal plane, the midbrain has a characteristic butterfly shape. The Substantia Nigra are thin structures located at the front of both hemispheres. A hemisphere-specific coordinate system is computed to express voxel spatial location accounting for inter-patient asymmetric changes of scales and orientation.

reaches a leaf, and to use the stored posterior distribution. Considering a random forest consisting of $T$ trees, predictions can be simply computed by averaging tree posteriors: $P(\mathcal{E} \mid \mathbf{x}, \mathbf{I})=\frac{1}{T} \sum_{t}^{T} P_{t}(\mathcal{E} \mid \mathbf{x}, \mathbf{I})$.

Learning the Prior $\boldsymbol{P}(\mathcal{S} \mid \mathbf{x})$ : As shown on Fig, 2 the midbrain has a characteristic butterfly shape in the transversal plane, which does not vary much along the longitudinal axis. The Substantia Nigra are thin structures located at the front of both hemispheres and do not vary much along the longitudinal axis either. Hence, we propose to express the location of each voxel using patient-specific coordinate systems that represent the left and right midbrain hemispheres in the transversal plane. By doing so, we can easily account for asymmetric changes of scales and orientation of the midbrain anatomy, which can occur in TCUS imaging. Let us denote by $\left\{\mathbf{x}_{m}\right\}_{m=1}^{M}=\mathcal{M}$, the finite set of $M$ voxels belonging to the midbrain. First, the centers of the left and right hemispheres are computed by performing a K-means clustering on $\mathcal{M}$. Then, each voxel is associated to its nearest cluster center to create the 2 hemisphere subsets $\mathcal{H}^{\text {left }}$ and $\mathcal{H}^{\text {right }}$. Finally, principal component analysis is applied to each of these subsets to compute a hemisphere-specific transversal coordinate system, and the location of each point is expressed in the normalized coordinate systems of the hemisphere it belongs to. The in-plane location of each voxel $\mathbf{x}_{m}$ can then be encoded by a vector $\mathbf{x}_{m}^{\prime}=\left[x_{m}^{\prime}, y_{m}^{\prime}, h_{m}\right]$, where $x_{m}^{\prime}$ and $y_{m}^{\prime}$ are the in-plane components in the hemisphere coordinate system, and $h_{m}$ is a categorical variable encoding the left/right side. To summarize, each voxel $\mathbf{x}_{m}$ is associated for the training phase to a couple $\left(\mathbf{x}^{\prime}{ }_{m}, \mathcal{S}_{m}\right)$, where $\mathcal{S}_{m}$ is equal to 1 if $\mathbf{x}_{m}$ belongs to the Substantia Nigra and 0 if not. As in the previous section, we use a random forest to learn the prior $P(\mathcal{S} \mid \mathbf{x})$ using a training set of 3D-TCUS from different patients. During the training, each tree aims at separating the SN from the rest of the midbrain, and creates clusters in its leaves that are consistent in terms of spatial location $\mathbf{x}_{m}^{\prime}$. 

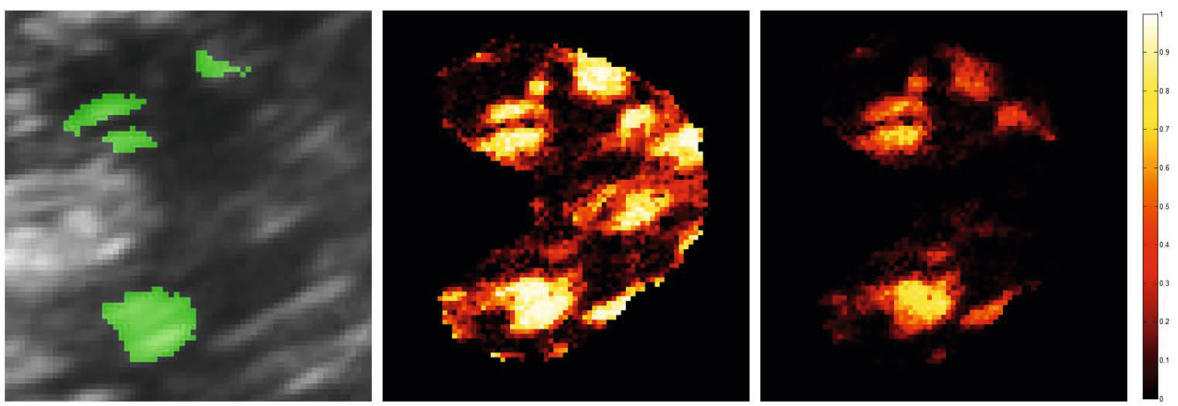

Fig. 3. The effect of our spatial prior: From left to right, (i) the manual segmentation overlayed on the US data, (ii) the predicted posterior using the data term forest and (iii) the output after combining with the forest-based spatial prior. All outputs are probabilistic and can be thresholded to provide a binary segmentation.

SNE Detection: Once the data term and the prior have been learned from a set of labelled midbrains, a new unseen patient data can be processed as follows: (1) the midbrain is segmented, (2) the hemisphere coordinate systems are determined using K-means followed by a PCA, (3) the probability $P(\mathcal{E} \mid \mathbf{x}, \mathbf{I})$ and the prior $P(\mathcal{S} \mid \mathbf{x})$ are computed for each voxel, and (4) the joint probability $P(\mathcal{E}, \mathcal{S} \mid \mathbf{x}, \mathbf{I})$ can be predicted using Eq1. Hence, we obtain for each voxel a probability of belonging to an SNE, and we can use a threshold $\mathcal{T} \in[0,1]$ to create a binary segmentation of the ferrite deposits: $\mathbf{c}=1$ if $P(\mathcal{E}, \mathcal{S} \mid \mathbf{x}, \mathbf{I}) \geq \mathcal{T}$, and $\mathbf{c}=0$ otherwise.

\section{$3 \quad$ Experiments and Results}

In this section, we evaluate our SNE detection approach on the bi-lateral 3DTCUS dataset volume of 22 subjects, consisting of 11 PD patients and 11 healthy controls. The $3 \mathrm{D}$ volumes were reconstructed at an isotropic resolution of $0.45 \mathrm{~mm}$ and labelled by a blinded expert into the regions "midbrain", "SNE left" and "SNE right". For our validation, we will consider this labeling as gold standard. We conduct comparative experiments to evaluate our SNE detection approach based on 2 discriminative models (VisForest-PriorForest) against the simple forest without spatial prior (VisForest), and a forest with a spatial prior constructed using a Gaussian distribution model for each hemisphere (VisForestGaussianPrior). The parameters of each Gaussian spatial prior are estimated by computing the sample mean and the covariance of the location of the SNE voxels in their hemisphere coordinate systems.

We perform a leave-one-patient-out cross-validation (LOO), i.e. we train all models on 21 labelled midbrains and test on the remaining one. As the outputs from our system are probabilities between 0 and 1, we perform a ROC analysis, i.e. we vary the threshold's value to compute a binary segmentation, compute the corresponding confusion matrices for each run and derive different quality 
Table 1. Overall SNE Detection results on 22 patients: The proposed prior permits to achieve better detecton by improving the specificity, i.e. by better rejecting echogenicities that do not belong to the estimated SN. Moreover, using a forest-based prior provides slightly better results.

\begin{tabular}{|c|c|c|c|c|c|c|c|c|c|}
\hline & \multicolumn{3}{|c|}{ F-measure } & \multicolumn{3}{c|}{ Specificity } & \multicolumn{3}{c|}{ Sensitivity } \\
\hline & Mean & Std & Median & Mean & Std & Median & Mean & Std & Median \\
\hline VisForest & 0.456 & 0.115 & 0.463 & 0.775 & 0.060 & 0.779 & $\mathbf{0 . 8 4 5}$ & $\mathbf{0 . 0 8 1}$ & $\mathbf{0 . 8 5 9}$ \\
\hline VisForest-GaussianPrior & 0.508 & 0.155 & 0.547 & 0.819 & 0.045 & 0.812 & 0.829 & 0.113 & 0.844 \\
\hline VisForest-PriorForest & $\mathbf{0 . 5 1 9}$ & $\mathbf{0 . 1 4 8}$ & $\mathbf{0 . 5 7 4}$ & $\mathbf{0 . 8 3 5}$ & $\mathbf{0 . 0 4 3}$ & $\mathbf{0 . 8 3 2}$ & 0.828 & 0.099 & 0.829 \\
\hline
\end{tabular}

measures: f-measure, specificity and sensitivity. While the number of trees is set to 10 for all experiments, the depth parameter is tuned by doing a discrete search (i.e. depth $=5,10,15,20$ ) and performing a full LOO for each depth value. Best results were obtained for a depth $=15$ for the VisForest, and for a depth $=10$ for the PriorForest.

Overall results are presented in Tab. 1. On the left, the best f-measure are reported by using threshold values of $0.5,0.1$ and 0.2 respectively for the VisForest, VisForest-GaussianPrior and VisForest-PriorForest models. By including our hemisphere-specific spatial prior, the f-measure is increased from $\mathbf{0 . 4 5 6}$ (VisForest) to $\mathbf{0 . 5 1 8}$ (VisForest-PriorForest). Moreover, learning this prior distribution using a random forest provides slightly better results than with Gaussian prior achieving $\mathbf{0 . 5 0 8}$. On the right, the best compromise between sensitivity and specificity are computed from the ROC analysis for all approaches. As illustrated by Fig. 3, the proposed prior permits to achieve improved specificity by better rejecting echogenicities that do not belong to the estimated SN. By varying the segmentation threshold, we also compute the area under curve which is $\mathbf{A U C}=\mathbf{0 . 9 0 3}$ for our approach, compared to a VisForest alone $\mathbf{A U C}=\mathbf{0 . 8 7 9}$ or with a simple Gaussian prior $\mathbf{A U C}=\mathbf{0 . 8 9 1}$.

\section{Discussion and Conclusion}

In this paper, we presented the first approach for the automatic detection of Substantia Nigra Echogenicities in 3D-TCUS. As the interpretation of such data is very difficult and yields high inter and intra-observer variability, our aim is to provide an objective and reliable segmentation of such Parkinson-related speckle patches. Inspired by the way medical experts recognize SNE, we proposed a probabilistic formulation combining two discriminative models: (1) a "visual" random forest specialized on the detection of echogenicities and (2) a "spatial" random forest modeling a location prior within the midbrain. Therefore, voxel locations are parametrized within hemisphere-specific coordinate systems in order to account for asymmetric changes of orientation and scale in the midbrain anatomy. In experimentations conducted on 22 patients data, we could assess the reliability of our approach that achieves a sensitivity and specificity of around $\mathbf{8 3 \%}$. From the segmentation output of our system, we can quantify automatically the 
amount of hyper-echogenicities in each hemisphere, with the motivation of using this information within a computer aided diagnosis system for Parkinson disease based on 3D-TCUS in the near future.

\section{References}

1. Ahmadi, S.-A., Baust, M., Karamalis, A., Plate, A., Boetzel, K., Klein, T., Navab, N.: Midbrain Segmentation in Transcranial 3D Ultrasound for Parkinson Diagnosis. In: Fichtinger, G., Martel, A., Peters, T. (eds.) MICCAI 2011, Part III. LNCS, vol. 6893, pp. 362-369. Springer, Heidelberg (2011)

2. Berg, D., Seppi, K., Behnke, S., Liepelt, I., Schweitzer, K., Stockner, H., Wollenweber, F., Gaenslen, A., Mahlknecht, P., Spiegel, J., Godau, J., Huber, H., Srulijes, K., Kiechl, S., Bentele, M., Gasperi, A., Schubert, T., Hiry, T., Probst, M., Schneider, V., Klenk, J., Sawires, M., Willeit, J., Maetzler, W., Fassbender, K., Gasser, T., Poewe, W.: Enlarged substantia nigra hyperechogenicity and risk for Parkinson disease: a 37-month 3-center study of 1847 older persons. Arch. Neurol. 68, 932-937 (2011)

3. Breiman, L.: Random forests. Machine Learning 45(1), 5-32 (2001)

4. Chen, L., Seidel, G., Mertins, A.: Multiple Feature Extraction for Early Parkinson Risk Assessment Based on Transcranial Sonography Image. In: IEEE Int. Conf. on Image Processing (2010)

5. Criminisi, A., Shotton, J., Konukoglu, E.: Decision forests: A unified framework for classification, regression, density estimation, manifold learning and semi-supervised learning. In: Foundations and Trends in Computer Graphics and Vision (2012)

6. Engel, K., Toennis, K.D.: Segmentation of the Midbrain in Transcranial Sonographies using a Two-Component Deformable Model. Annals of the British Machine Vision Association and Society for Pattern Recognition (BMVA) 4, 1-12 (2009)

7. Gaenslen, A., Unmuth, B., Godau, J., Liepelt, I., Di Santo, A., Schweitzer, K.J., Gasser, T., Machulla, H.J., Reimold, M., Marek, K., Berg, D.: The specificity and sensitivity of transcranial ultrasound in the differential diagnosis of Parkinson's disease: a prospective blinded study. Lancet Neurol. 7, 417-424 (2008)

8. Geremia, E., Menze, B.H., Clatz, O., Konukoglu, E., Criminisi, A., Ayache, N.: Spatial Decision Forests for MS Lesion Segmentation in Multi-Channel MR Images. In: Jiang, T., Navab, N., Pluim, J.P.W., Viergever, M.A. (eds.) MICCAI 2010, Part I. LNCS, vol. 6361, pp. 111-118. Springer, Heidelberg (2010)

9. Kier, C., Cyrus, C., Seidel, G., Hofmann, U.G., Aach, T.: Segmenting the substantia nigra in ultrasound images for early diagnosis of Parkinson's disease. Int. J. of Computer Assisted Radiology and Surgery 2(S1), S83-S85 (2007)

10. Pauly, O., Glocker, B., Criminisi, A., Mateus, D., Möller, A.M., Nekolla, S., Navab, N.: Fast Multiple Organ Detection and Localization in Whole-Body MR Dixon Sequences. In: Fichtinger, G., Martel, A., Peters, T. (eds.) MICCAI 2011, Part III. LNCS, vol. 6893, pp. 239-247. Springer, Heidelberg (2011)

11. Vlaar, A., de Nijs, T., van Kroonenburgh, M., Mess, W., Winogrodzka, A., Tromp, S., Weber, W.: The predictive value of transcranial duplex sonography for the clinical diagnosis in undiagnosed Parkinsonian syndromes: comparison with SPECT scans. BioMed Central Neurology 8, 42 (2008)

12. Walter, U., Dressler, D., Probst, T., Wolters, A., Abu-Mugheisib, M., Wittstock, M., Benecke, R.: Transcranial brain sonography findings in discriminating between parkinsonism and idiopathic Parkinson disease. Arch. Neurol. 64(11), 1635-1640 (2008) 